\title{
Anemia e renda per capita familiar de crianças freqüentadoras da creche do Centro Educacional Unificado Cidade Dutra, no Município de São Paulo
}

Anemia and per capita income in children enrolled in a childhood education center in São Paulo, Brazil

Teresinha Stumpf Souto ${ }^{1}$, Mariana de Novaes Oliveira², Flávio Casoy ${ }^{3}$, Edna Helena S. Machado ${ }^{4}$, Yara Juliano ${ }^{5}$,

Lélia Cardamone Gouvêa ${ }^{6}$, Jane Eston Armond

RESUMO

Objetivo: Avaliar a freqüência de anemia de acordo com a renda familiar per capita de crianças matriculadas no Centro de Educação Infantil (CEI) do Centro Educacional Unificado (CEU) Cidade Dutra, no Município de São Paulo, São Paulo, em 2004.

Métodos: Estudo transversal, com 190 crianças de 11 a 57 meses de idade distribuídas em duas faixas de renda familiar per capita ( $<1$ e $\geq 1$ salário mínimo). Foram consideradas anêmicas as crianças com taxa de hemoglobina $<11 \mathrm{~g} / \mathrm{dL}$, medida em amostra de sangue capilar digital por meio de fotômetro portátil Hemocue ${ }^{\circledR}$. Na análise dos resultados foram empregados o teste do qui-quadrado e a análise de variância de Kruskal-Wallis.

Resultados: A anemia foi observada em 31,6\% das crianças investigadas, sendo a sua freqüência significantemente maior nas crianças de menor faixa etária. A freqüência de anemia foi de $72,7 \%, 41,2 \%, 19,7 \%$ e 15,4\%, respectivamente nas crianças de 11 a 23 meses, 24 a 35 meses, 36 a 47 meses e 48 a 57 meses. Entre as crianças de famílias com renda inferior a um salário mínimo, a freqüência de anemia foi de $36,7 \%$, e naquelas com um ou mais salário mínimo, a prevalência foi $22,9 \%$.

Conclusões: Na creche do CEU Cidade Dutra, a freqüência de anemia foi maior nas crianças de 11 a 23 meses e nas famílias com renda per capita inferior a um salário mínimo.

Palavras-chaves: anemia; criança; renda per capita.

\section{ABSTRACT}

Objective: To evaluate the frequency of anemia according to family per capita income in children enrolled in a day care center in São Paulo, Brazil, in 2004.

Methods: This cross-sectional study enrolled 190 children aged 11 to 57 months divided into two groups of per capita income ( $<1$ or $\geq 1$ minimum wage). Hemoglobin level was evaluated in capillary blood (finger-stick test) using a Hemocue ${ }^{\circledR}$ portable photometer. Anemia was considered when hemoglobin level was below $11 \mathrm{~g} / \mathrm{dL}$. The results were analyzed using chi-square and Kruskal-Wallis tests.

Results: Anemia was found in $31.6 \%$ of the investigated children, with a higher proportion in younger children. The frequency of anemia was, respectively, $72.7 \%, 41.2 \%$, $19.7 \%$ and $15.4 \%$ in children aged 11-23 months age, 24-35 months, 36-47 months, and 48-57 months. Among children of families with low per capita income, the frequency of anemia was $36.7 \%$. In families earning more than one minimum wage per capita per month, there were $22.9 \%$ of children with anemia.

Conclusions: At the day care center, the frequency of anemia was higher among children aged 11-23 months, especially when the family per capita income was less than one minimum wage per month.

Key-words: anemia, child; per capita income.
'Pediatra da Prefeitura Municipal de São Paulo e mestre em Ciências pela Universidade Federal de São Paulo (Unifesp-EPM)

${ }^{2}$ Nutricionista e mestre em Ciências da Saúde pela Unifesp-EPM

${ }^{3}$ Estudante de Medicina da Brown University Medical School, Providence, RI, USA

${ }^{4}$ Nutricionista da Secretaria Municipal da Saúde de São Paulo e mestre em Ciências pela Unifesp-EPM

${ }^{5}$ Professora doutora de Bioestatística da Universidade de Santo Amaro (Unisa) 'Professora titular de Pediatria da Unisa, responsável pelo Ambulatório de Aleitamento Materno da Disciplina de Nutrologia do Departamento de Pediatria da Unifesp-EPM e assessora técnica da saúde da criança da Coordenadoria de Saúde da Capela do Socorro da Secretaria Municipal de Saúde
${ }^{7}$ Professora doutora em Saúde Pública pela Universidade de São Paulo (USP), docente da disciplina de Epidemiologia da Faculdade de Medicina da Unisa e Coordenadora de Saúde da Capela do Socorro

Endereço para correspondência:

Teresinha Stumpf Souto

Rua João Moura, 1.310, apto.32

CEP 05412-003 - São Paulo/SP

E-mail: teresouto@terra.com.br

Recebido em: 17/10/2006

Aprovado em: 4/4/2007 


\section{Introdução}

O grande desafio nutricional a ser vencido atualmente é o da anemia ferropriva. A anemia por deficiência de ferro é a desordem nutricional mais comum no mundo, afetando populações tanto em países desenvolvidos como em desenvolvimento ${ }^{(1-8)}$.

Estima-se que $25 \%$ da população mundial apresente carência de ferro ${ }^{(1,9,10)}$. A Organização Mundial de Saúde (OMS) considera que cerca da metade das crianças menores de cinco anos nos países em desenvolvimento sofram de anemia. No Brasil, a freqüência de casos vem aumentando e já é considerada a carência nutricional mais prevalente, presente em cerca de $50 \%$ das crianças de seis meses a seis anos ${ }^{(11,12)}$.

Entre os determinantes da anemia em crianças se encontram: baixo nível socioeconômico, baixo peso ao nascimento, desmame precoce, introdução precoce do leite de vaca e ingestão insuficiente de ferro em crianças menores de dois anos ${ }^{(3,6,13,14)}$.

Independentemente das causas que determinam o estado anêmico, este pode se associar a prejuízos no desenvolvimento cognitivo e motor da criança e no aproveitamento escolar no futuro ${ }^{(3,4,8,15-19)}$.

Devido ao baixo poder aquisitivo, muitas famílias de regiões periféricas no Município de São Paulo sobrevivem com renda irregular e inferior a um salário mínimo. Em tais situações, inviabiliza-se o acesso à alimentação quantitativa e/ou qualitativamente adequada, com grande risco de deficiências nutricionais.

Cada vez mais as mulheres estão ingressando no mercado de trabalho para aumentar a renda familiar e, portanto, necessitam de local apropriado para deixarem seus filhos. As creches surgiram no início do século XX com o objetivo de combater a pobreza, a desnutrição e a mortalidade infantil, bem como atender às necessidades das mulheres que trabalhavam fora de casa ${ }^{(20)}$. Concebidas como um local complementar ao cuidado da família, as creches devem oferecer um contexto favorável à promoção do desenvolvimento infantil, além da educação da criança ${ }^{(21,22)}$. No ano de 2003, o Instituto Nacional de Estudos e Pesquisas Educacionais (Inep) informou que foram atendidas mais de 766 mil crianças em creches municipais e estaduais no país. Somente no Estado de São Paulo havia aproximadamente 186 mil crianças matriculadas ${ }^{(23)}$.
Neste mesmo ano, em São Paulo, foram criados os Centros Educacionais Unificados (CEUS), escolas situadas em regiões da periferia da cidade, conhecidas pela exclusão social. O CEU Cidade Dutra foi inaugurado em agosto de 2003, no distrito administrativo de Cidade Dutra, na Subprefeitura da Capela do Socorro, região Sul do Município de São Paulo. A Subprefeitura da Capela do Socorro é formada por três distritos administrativos: Socorro, Cidade Dutra e Grajaú. Possui área de 134,2 km km $^{(24)}$ e 619.644 habitantes, sendo a mais populosa da região Sul de São Paulo. Esta região da cidade de São Paulo é composta pelas subprefeituras de M’Boi Mirim, Campo Limpo, Santo Amaro, Capela do Socorro e Parelheiros. A subprefeitura da Capela do Socorro apresenta o maior número de nascidos vivos no Município de São Paulo e a subprefeitura de M'Boi Mirim é a segunda colocada na mesma classificação ${ }^{(25-27)}$.

Em abril de 2004, data de início da coleta de dados desta pesquisa, eram atendidas cerca de 300 crianças de até quatro anos e 11 meses de idade, que recebiam até cinco refeições diárias e que permaneciam na creche do CEU Cidade Dutra por períodos de até 12 horas. Como a anemia se associa ao baixo nível socioeconômico e sua maior prevalência ocorre em crianças menores de cinco anos de idade, este trabalho teve como objetivo avaliar a presença de anemia e sua relação com a renda per capita das famílias entre crianças matriculadas no Centro de Educação Infantil do CEU Cidade Dutra.

\section{Métodos}

Estudo transversal, descritivo e analítico. O projeto de pesquisa foi analisado e aprovado pelo Comitê de Ética e Pesquisa da Universidade de Santo Amaro (Unisa).

Após o contato preliminar com a gestora do CEU e a diretora da creche, realizou-se reunião com as educadoras para explicitação do Projeto, seus objetivos e sua viabilidade. Em outro momento, todos os pais foram convidados a participar de reunião para dirimir as dúvidas, entregar carta explicativa e agendar horários para o preenchimento dos questionários. Os pais que desejaram participar compareceram nas datas agendadas, ocasião em que assinaram o Termo de Livre Consentimento Esclarecido e responderam, a seguir, ao questionário aplicado por dois pesquisadores devidamente treinados. 
Não foi realizada seleção das crianças estudadas, pois a pretensão do trabalho era a de englobar todo o universo das crianças da creche. Das 290 crianças matriculadas, 34 mães ou responsáveis não tiveram interesse em participar da pesquisa, cinco não responderam ao questionário, 12 crianças abandonaram a creche no período do estudo e 49 estavam ausentes da creche nos dias de coleta de sangue. Dessa forma, foram incluídas na pesquisa 190 crianças. Dentre as 49 crianças ausentes no dia da coleta de sangue, estavam as 12 crianças menores que 11 meses de idade matriculadas na creche.

As crianças participantes do estudo foram separadas em grupos segundo a idade: 11 a 23 meses, 24 a 35 meses, 36 a 47 meses, e 48 a 57 meses.

Para a dosagem dos níveis de hemoglobina, foi utilizado o hemoglobinômetro digital portátil de marca HemoCue $-\beta$ Hemoglobin Photometer ${ }^{\circledR}$, cujo princípio se baseia na leitura fotométrica com a utilização de microcuvetas $\beta$ hemoglobin. A punção digital foi realizada por um único pesquisador treinado, com o uso de lanceta descartável marca Easy Lets ${ }^{\circledR}$. A punção foi feita na polpa digital do quarto dedo da mão direita, sendo a gota de sangue coletada na microcuveta descartável e, em seguida, inserida no hemoglobinômetro para leitura.

Para a classificação da anemia, foi utilizado o ponto de corte de hemoglobina menor de $11 \mathrm{~g} / \mathrm{dL}$ para crianças de até 59 meses, como o preconizado pela $\mathrm{OMS}^{(28)}$.

As informações sobre a idade da criança e a renda familiar foram coletadas durante a entrevista agendada com a mãe ou responsável. Em relação à renda per capita, o valor informado foi dividido pelo número de pessoas que dependiam financeiramente dessa renda e ajustado de acordo com o salário mínimo vigente na época: trezentos reais ( $\mathrm{R} \$ 300,00)$. Constituíram-se dois grupos: grupo I (menos de um salário mínimo per capita) e grupo II (um ou mais salários mínimos per capita).
Para a análise estatística, foram aplicados os seguintes testes: análise de variância de Kruskal-Wallis ${ }^{(29)}$, para comparar os níveis de hemoglobina para as faixas etárias de ambos os gêneros; teste do qui-quadrado ${ }^{(29)}$, para estudar possíveis associações entre presença ou ausência de anemia nas variáveis estudadas. Fixou-se em 0,05 ou $5 \%$ o nível de rejeição da hipótese de nulidade.

\section{Resultados}

As 190 crianças que participaram do estudo apresentavam idade entre 11 e 57 meses. A distribuição por gênero foi de $90(48,4 \%)$ para o masculino e $100(51,6 \%)$ para o feminino.

Quanto ao grupo etário, 33 crianças tinham de 11 a 23 meses de idade; 35 de 24 a 35 meses; 70 de 36 a 47 meses e, no grupo de 48 a 57 meses, havia 52 crianças.

A anemia foi constatada em $31,6 \%$ das crianças estudadas. A freqüência de anemia diminuiu com o aumento da faixa etária. Observou-se que a freqüência de anemia nas crianças do grupo etário de 11 a 23 meses foi significantemente maior que a encontrada nas outras faixas etárias (Tabela 1).

Os menores valores médios da hemoglobina foram encontrados no grupo etário de 11 a 23 meses (Tabela 2). A análise de variância de Kruskal-Wallis mostrou que, tanto para o gênero masculino quanto para o feminino, o grupo etário de 11 a 23 meses apresentou valores de hemoglobina menores do que o grupo de 36 meses ou mais. O grupo etário de 24 a 35 meses não apresentou diferença significante em relação aos demais.

Em relação à renda familiar, a freqüência da anemia foi significantemente maior entre as crianças oriundas de famílias com renda per capita inferior a um salário mínimo, comparadas àquelas provenientes de famílias com renda igual ou superior a um salário mínimo (Tabela 3).

Tabela 1 - Freqüência de anemia nas crianças matriculadas no Centro Educacional Unificado Cidade Dutra, segundo o grupo etário

\begin{tabular}{lcccc}
\hline Grupo etário & Anemia presente & Anemia ausente & Total & $\%$ anêmicos \\
\hline $11-23$ meses & 24 & 9 & 33 & 72,7 \\
$24-35$ meses & 14 & 21 & 35 & 41,2 \\
$36-47$ meses & 14 & 56 & 70 & 19,7 \\
$48-57$ meses & 8 & 44 & 52 & 15,4 \\
Total & 60 & 130 & 190 & 31,6 \\
\hline
\end{tabular}

Qui-quadrado calculado $=37,66 ; p<0,0001$ 


\section{Discussão}

O modelo do estudo buscou analisar as relações entre a anemia em crianças matriculadas no Centro de Educação Infantil do CEU Cidade Dutra e a renda per capita de suas famílias.

A frequiência de anemia encontrada neste estudo foi semelhante em ambos os gêneros, como relatado por outros autores $^{(4,30)}$.

Entre as crianças de 11 a 57 meses, a freqüência de anemia observada neste estudo também foi semelhante aos valores descritos na literatura. Em Ribeirão Preto, São Paulo, Dutra de Oliveira et al ${ }^{(31)}$ encontraram, em crianças de creche de dois a seis anos de idade, uma prevalência de anemia de $58 \%$. Já no estudo realizado por de Almeida et $a^{l^{(30)}}$ em 503 crianças de 12 a 83,9 meses de idade de nove creches na cidade de Monte Alto, a freqüência de anemia foi $14,5 \%$.

A análise da freqüência de anemia segundo o grupo etário mostrou que esta foi maior em crianças de 11 a 23 meses, sendo que nesse grupo etário aproximadamente 3/4 das crianças apresentaram valores de hemoglobina inferiores a $11 \mathrm{~g} / \mathrm{dL}$. A ocorrência da anemia diminuiu nos grupos etários maiores. A alta prevalência de anemia encontrada na população de lactentes foi coincidente com a literatura ${ }^{(19,32,33)}$.

Machado et $a l^{(19)}$ analisaram a taxa de hemoglobina de 942 crianças em oito creches beneficentes do Município de São Paulo e encontraram freqüência de anemia de 64,5\% na faixa etária de 13 a 24 meses. Brunken et al ${ }^{(32)}$, na cidade de Cuiabá, Mato Grosso, avaliaram 271 crianças de oito creches e observaram maior prevalência de anemia em crianças com menos de 24 meses de idade. Ling et al ${ }^{(33)}$ também encontraram associação estatisticamente significante entre as variáveis menor idade e anemia em crianças de creches públicas e filantrópicas no Município de São Paulo.

No lactente, devido à rápida mobilização das reservas que ocorre para acompanhar a elevada velocidade de crescimento, os mecanismos de regulação do balanço de ferro podem ser insuficientes. Aliada a isso, a maior suscetibilidade à anemia em crianças menores de 24 meses tem sido atribuída a fatores como desmame precoce com uso de leite de vaca integral, levando à micro-hemorragias intestinais e atraso na introdução de alimentos ricos em ferro de boa biodisponibilidade ${ }^{(34)}$.

Tabela 2 - Níveis de hemoglobina nas crianças matriculadas no Centro Educacional Unificado Cidade Dutra, segundo gênero e grupo etário

\begin{tabular}{lllllll}
\hline & $\begin{array}{l}\text { Hemoglobina } \\
(\mathbf{g} / \mathbf{d L})\end{array}$ & $\begin{array}{l}\mathbf{1 1 - 2 3} \\
\text { meses }\end{array}$ & $\begin{array}{l}\mathbf{2 4 - 3 5} \\
\text { meses }\end{array}$ & $\begin{array}{l}\mathbf{3 6 - 4 7} \\
\text { meses }\end{array}$ & $\begin{array}{l}\mathbf{4 8 - 5 7} \\
\text { meses }\end{array}$ & $\begin{array}{l}\text { Análise de variância } \\
\text { Kruskal-Wallis }\end{array}$ \\
\hline Masculino & Média & 10,4 & 11,4 & 12,1 & 12,4 & $\begin{array}{l}p<0,001 \\
11-23 \text { meses }<36-47 \text { meses e 48-57 meses } \\
p<0,001\end{array}$ \\
& Desvio padrão & 1,8 & 1,4 & 1,2 & 1,2 & $\begin{array}{l}11-23 \text { meses }<36-47 \text { meses e } 48-57 \text { meses } \\
p<0,001\end{array}$ \\
& Mediana & 10,2 & 11,6 & 11,9 & 12,6 & $\begin{array}{l}11-23 \text { meses }<36-47 \text { meses e } 48-57 \text { meses } \\
p<0,001\end{array}$ \\
& Média & 9,6 & 11,3 & 12,0 & 12,5 & $\begin{array}{l}11-23 \text { meses }<36-47 \text { meses e } 48-57 \text { meses } \\
p<0,001\end{array}$ \\
& Desvio padrão & 1,5 & 1,5 & 1,4 & 1,2 & $\begin{array}{l}11-23 \text { meses }<36-47 \text { meses e 48-57 meses } \\
p<0,001 \\
11-23 \text { meses }<36-47 \text { meses e 48-57 meses }\end{array}$ \\
\hline
\end{tabular}

Tabela 3 - Valores de hemoglobina $(\mathrm{Hb})$ das crianças matriculadas no Centro Educacional Unificado Cidade Dutra segundo renda per capita

\begin{tabular}{|c|c|c|c|c|}
\hline & $\mathrm{Hb}<11,0 \mathrm{~g} / \mathrm{dL}$ & $H \geq 11,0 \mathrm{~g} / \mathrm{dL}$ & Total & $\%$ anemia \\
\hline <1 salário mínimo & 44 & 76 & 120 & 36,7 \\
\hline$\geq 1$ salário mínimo & 16 & 54 & 70 & 22,9 \\
\hline Total & 60 & 130 & 190 & 31,6 \\
\hline
\end{tabular}

Qui-quadrado calculado $=3,90 ; p<0,05$ 
Apesar do cardápio da creche não ter sido analisado, é possível que, por se tratar de um serviço terceirizado, a sua elaboração não fosse adequadamente supervisionada pelo nutricionista da empresa. Além disso, embora as fontes de ferro e de vitamina $C$ fizessem parte do consumo diário, a quantidade de tais elementos ingeridos pela criança poderia ser insuficiente, devido à menor capacidade gástrica das crianças pequenas.

A baixa renda familiar deve ser uma das causas de carências nutricionais, entre elas a anemia ferropriva, pois pode dificultar a aquisição de alimentos ricos em ferro de boa biodisponibilidade. No presente estudo, a renda per capita menor que um salário mínimo se associou significantemente à anemia. Tal associação entre anemia e menor renda também foi observada em outros estudos realizados no Brasil ${ }^{(3,4,18)}$.

Monteiro $e$ t $a l^{(4)}$ analisaram a tendência de aumento da anemia na infância na cidade de São Paulo, e verificaram que, nos estratos inferiores e intermediários de renda familiar per capita, a evolução dos indicadores de anemia era mais desfavorável do que a observada nos estratos de maior renda. Do mesmo modo, Neuman et al ${ }^{(3)}$ salientaram a importância dos fatores de riscos sociais, evidenciando a força da desigualdade social na prevalência da anemia.

Entre as carências nutricionais, a redução da anemia por deficiência de ferro representa um dos maiores desafios, porém, as estratégias utilizadas para a prevenção desta morbidade não têm sido eficazes.

O combate à anemia exige ações em curto prazo, pelo risco ao desenvolvimento intelectual e à saúde das crianças, e, diante desse quadro, três estratégias, isoladas ou combinadas, são recomendadas para o controle da deficiência nutricional de ferro: as ações educativas, a suplementação medicamentosa e a fortificação compulsória de alimentos utilizados amplamente na dieta infantil ${ }^{(3)}$.
As ações educativas junto às crianças, pais, professores e toda a comunidade são importantes não só quanto à orientação alimentar, como na prevenção da anemia desde a gestação ${ }^{(3,35)}$.

O uso profilático de sulfato ferroso por crianças menores de dois anos é preconizado pela Sociedade Brasileira de Pediatria (SBP), porém, a baixa aderência à norma mantém os altos índices de anemia encontrados ${ }^{(36)}$.

A fortificação de alimentos é mundialmente considerada como a solução mais prática e de melhor custo-benefício, principalmente para regiões nas quais prevalência de anemia ferropriva é grande ${ }^{(37)}$.

Neste estudo, o leite fornecido pela creche não atendia às recomendações da SBP para a alimentação de lactentes. A população de baixa renda ainda tem acesso limitado ao uso de fórmulas lácteas modificadas devido ao seu alto custo. Um passo, ainda que tímido, nesse sentido, foi a resolução mais recente da Agência Nacional de Vigilância Sanitária (Anvisa) sobre a fortificação de farinhas, que se tornou obrigatória a partir de junho de 2004. Entretanto, o consumo de produtos derivados de farinha por crianças menores de 24 meses é bastante restrito em termos de quantidade, tornando o leite o alimento indicado para ser fortificado e consumido por crianças dessa faixa etária ${ }^{(38)}$.

Essas recomendações, embora não resolvam definitivamente o problema da anemia em virtude de seus determinantes econômicos, podem reduzir os índices alarmantes de anemia em crianças freqüentadoras de creches e, assim, contribuir para a melhoria da sua saúde.

Concluí-se, neste estudo, que a freqüência de anemia foi maior em crianças de 11 a 23 meses, oriundas de famílias com renda per capita inferior a um salário mínimo, ressaltando a importância do seguimento ambulatorial de lactentes no sentido de detectar precocemente a anemia, sobretudo após a introdução da alimentação complementar. 


\section{Referências bibliográficas}

1. Queiroz SS, Torres MAA. Iron deficiency anemia in children. J Pediatr (Rio J) 2000;76:S298-304.

2. Berger J, Aguayo VM, Téllez W, Luján C, Traissac P, San Miguel JL. Weekly iron supplementation is as effective as 5 day per week iron supplementation in Bolivian school children living at high altitude. Eur J Clin Nutr 1997;51:381-6.

3. Neuman NA, Tanaka OU, Szarfarc SC, Guimarães PRV, Victora CG. Prevalência e fatores de risco para anemia no Sul do Brasil. Rev Saude Publica 2000;34:56-63.

4. Monteiro CA, Szarfarc SC, Mondini L. Tendência secular da anemia na infância na cidade de São Paulo (1984-1996). Rev Saude Publica 2000;34:S62-72.

5. Silva D. Caracterização de anemia ferropriva em crianças de dois grupamentos etários no Município de Viçosa. In: Abstract of the III World Congress of Pediatric Nutrition, Resumos, 2001, São Paulo: Sociedade Brasileira de Pediatria.

6. Levy-Costa RB, Monteiro CA. Consumo de leite de vaca e anemia na infância no Município de São Paulo. Rev Saude Publica 2004;38:797-803.

7. Coutinho GG, Goloni-Bertollo EM, Bertelli EC. Iron deficiency anemia in children: a challenge for public health and for society. São Paulo Med J 2005;123:88-92.

8. Lynch SR. The impact of iron fortification on nutritional anaemia. Best Pract Res Clin Haematol 2005;18:333-46.

9. Walter T, Hertrampf E, Pizarro F, Olivares M, Llaguno S, Letelier A et al. Effect of bovine-hemoglobin-fortified cookies on iron status of schoochildren: a nationwide program in Chile. Am J Clin Nutr 1993;57:190-4

10. Terao SM, Puccini RF, Silva EM, Pedroso GC, Silva NN. Prevalência de anemia em crianças residentes no município do Embu (São Paulo), 1996-7. Rev Paul Pediatr 2004;22:7-14.

11. Valente FL. Do combate à fome à segurança alimentar e nutricional: o direito à alimentação adequada. R Nutr 1997;10:20-36.

12. Batista-Filho M. Da fome à segurança alimentar: retrospecto e visão prospectiva. Cad Saude Publica 2003;19:872-3.

13. Silva LM, Giugliani ER, Aerts DR. Prevalência e determinantes de anemia em crianças de Porto Alegre, RS, Brasil. Rev Saude Publica 2001;35:66-73.

14. Brito LL, Barreto ML, Silva RC, Assis AM, Reis MG, Parraga I et al. Fatores de risco para anemia por deficiência de ferro em crianças e adolescentes parasitados por helmintos intestinais. Rev Panam Salud Pública 2003;14:422-31.

15. Souza SB, Szarfarc SC, Souza JM. Anemia no primeiro ano de vida em relação ao aleitamento materno. Rev Saude Publica 1997;31:15-20.

16. World Health Organization and the United Nations Children's Fund. Focusing on anaemia. Towards an integrated approach for effective anaemia control. WHO, Geneva, 2004. Disponível em: http://www.who.int/nutrition/publications/ WHOandUNICEF_statement_anaemia.pdf. Acessado em 12 de outubro de 2006.

17. Almeida CA, Ricco RG, Ciampo LA, Souza AM, Pinho AP, Oliveira JE. Factors associated with iron deficiency anemia in Brazilian preschool children. J Pediatr (Rio J) 2004;80:229-34.

18. Neves MB, Silva EM, Morais MB. Prevalência e fatores associados à deficiência de ferro em lactentes atendidos em um centro de saúde-escola em Belém, Pará, Brasil. Cad Saude Publica 2005;21:1911-8.

19. Machado EH, Brasil AL, Palma D, Taddei JA. Condição nutricional e prevalência de anemia em crianças matriculadas em creches beneficentes. Rev Paul Pediatria 2005;23:21-6.
20. Saraiva, JM. Perfil do trabalhador do setor de nutrição e saúde de creches públicas da cidade do Recife-PE [tese de mestrado]. Recife (PE): Universidade Federal de Pernambuco; 1999.

21. Rossetti-Ferreira MC, Amorim KS, Vitória T. A creche enquanto contexto possível de desenvolvimento da criança pequena. Rev Bras Cresc Desenv Hum 1994;4:35-40.

22. Rizzo G. Creche: organização, currículo, montagem e funcionamento. Rio de Janeiro: Berthrand Brasil; 2000.

23. INEP - Instituto Nacional de Pesquisas Educacionais. Censo Escolar 2003. Brasília. Disponível em: http://www.inep.gov.br/download/estatisticas/sinopse_estatisticas_2003/censo-miolo1-2003.pdf. Acessado em 12 de outubro de 2006.

24. IBGE - Instituto Brasileiro de Geografia e Estatística. Estimativas de população [homepage na Internet]. Citado em 12 junho de 2006. Disponível em: http://www.ibge.gov.br

25. São Paulo. Secretaria Municipal de Saúde. Serviço de Informação de Nascimentos. São Paulo: SINASC; 2004. Disponível em: http://ww2.prefeitura. sp.gov.br//arquivos/secretarias/saude/publicacoes/0004/BoletimSINASC.pdf

26. São Paulo. Prefeitura da Cidade de São Paulo. Subprefeituras. 2007. Disponível em: http://www.capital.sp.gov.br/portalpmsp/do/orgaos?op=viewForm\& coTipoUnidade. Acessado em 12 de outubro de 2006.

27. Sposati A. Mapa da exclusão/inclusão social da cidade de São Paulo. Núcleo de Estudos e Pesquisas sobre seguridade e Assistência Social da PUC/SP $1^{\text {a }}$ ed. São Paulo: PUC; 2001.

28. World Health Organization. Iron deficiency anaemia: assessment, prevention, and control: a guide for programme managers. WHO/NDH/01.3. Geneve: WHO; 2001

29. Siegel Jr S, Castellan NJ. Estatística não paramétrica para ciências do comportamento. $2^{a}$ ed. Porto Alegre: Artmed; 2006.

30. Almeida CA, Baptista ME, Crott GC, Cantaloni A, Ricco RG, Del Ciampo LA et al. Monte Alto sem anemia, primeira fase: avaliação nutricional das crianças pré-escolares. Rev Paul Pediatria 2002,20:275-9.

31. Dutra de Oliveira JE, Ferreira JB, Vasconcellos VP, Marchini JS. Drinking water as an iron carrier to control anemia in preschool children in a day-care center. J Am Coll Nutr. 1994;13:198-202.

32. Brunken GS, Guimarães LV, Fisberg M. Anemia em crianças menores de 3 anos que freqüentam creches públicas em período integral. J Pediatr (Rio J) 2002,78:50-6.

33. Ling NT, Ribeiro LC, Konstantyner T, Taddei JA. Anemia em lactentes que freqüentam creches públicas e filantrópicas no Município de São Paulo. Rev Paul Pediatria 2006, 24:330-4.

34. Ziegler EE, Jiang T, Romero E, Vinco A, Frantz JA, Nelson SE. Cow's milk and intestinal blood loss in late infancy. J Pediatr 1999;135:720-6.

35. Szarfarc SC. Diagnóstico de deficiência de ferro na infância. Rev Saude Publica 1985;19:278-84

36. Sociedade Brasileira de Pediatria. Departamento de Nutrologia. Manual de orientação: alimentação do lactente, alimentação do pré-escolar, alimentação do escolar, alimentação do adolescente, alimentação na escola. São Paulo: Sociedade Brasileira de Pediatria. Departamento de Nutrologia, 2006.

37. Zlotkin SH, Schauer C, Christofides A, Sharieff W, Tondeur MC, Hyder SM Micronutrient sprinkles to control childhood anaemia. PLoS Med. 2005;2:e1.

38. Brasil, Ministério da Saúde. ANVISA aprova o regulamento técnico para a fortificação. D.O.U. (Diário Oficial da União); Poder Executivo. Brasília Resolução - RDC no344, de 13 de dezembro de 2002. Disponível em: http://www. anvisa.gov.br/alimentos/farinha.htm 